Pacific Journal of Mathematics

SETS WHICH CAN BE MISSED BY SIDE APPROXIMATIONS 


\title{
SETS WHICH CAN BE MISSED BY SIDE APPROXIMATIONS TO SPHERES
}

\author{
J. W. CANNON
}

In this paper it is proved that a 2-sphere $S$ in $E^{3}$ can be side approximated from Int $S$ so as to miss a closed subset $X$ of $S$ if and only if $(S \cup \operatorname{Int} S)-X$ is $1-$-ULC.

If $X=\{p\}$ is a singleton, then the 1-ULC property described in the first paragraph reduces to a property studied by McMillan [22] in connection with piercing points $p$ of a crumpled cube (see (0.2) below). Our work was motivated by his paper although our methods bear little relationship to his.

Our result has as immediate corollaries a number of important, well-known theorems, four of which we now mention:

(0.1) ([2. Th. 2]). A 2-sphere $S$ in $E^{3}$ is tame if $E^{3}-S$ is 1-ULC.

(0.2) ([22, Th. 1]). If $C$ is a crumpled cube in $E^{3}$ and $p \in \operatorname{Bd} C$, then $p$ is a piercing point of $C$ if and only if $C-p$ is 1-ULC.

(0.3) ([19, Th. 3]). If $S$ and $S^{\prime}$ are 2-spheres in $E^{3}$ and $X \subset U \subset$ $S \cap S^{\prime}$, where $X$ is compact and $U$ is open in $S$ and $S^{\prime}$. Then $\left(*, X, S^{\prime}\right)$ is satisfied if and only if $(*, X, S)$ is satisfied.

(0.4) ([8] and [10]). If $S$ is a 2-sphere in $E^{3}, X$ is a compact subset of $S$, and $X=\bigcup_{i=1}^{\infty} X_{i}$, where each $X_{i}$ is compact and satisfies $\left(*, X_{i}, S\right)$, then $(*, X, S)$ is satisfied.

Properties similar to the 1-ULC property described in the first paragraph have been studied previously (e.g., properties $(A, F, S)$ and $(B, F, S)$ in [19]). However, the properties studied have, in general, had only restricted application to the question of determining which subsets of a 2-sphere can be missed by side approximations to that sphere and thus have been inapplicable in results such as $(0.2),(0.3)$, and (0.4).

Precise definitions appear in $\S 1$. Our main result, mentioned in the first paragraph, is proved in $\S 2$. We also present in $\S 2$ a slightly strengthened version of our main result for use in another paper (Lemma 2.5 and Theorem 2.6). The four corollaries mentioned above are proved in $\S 3$. In $\S 3$ we also remark on ways in which our main result can be used to sharpen other results which have appeared in the literature.

The part of this paper actually necessary for the proof of Theorem (0.1) appears in five short paragraphs. This proof is considerably shorter and conceptually easier to follow than the original proof. Our proof of Theorem (0.3) is likewise much shorter than the original. The 
great simplifying factor is the use of Lister's form [18, Th. 3] of Bing's Side Approximation Theorem; Lister's theorem can be used to simplify the proof of many other results on the embeddings of surfaces in 3-manifolds (cf. [12]). Except for Lister's theorem, the tools we have chosen to use are essentially those which were used in the original proofs of Theorems (0.1)-(0.4). Lemma 2.5 and Theorem 2.6 are slightly more complex than the other results.

We had not originally planned to restrict ourselves in this paper to the applications of our main theorem which appear in $\S 3$ (cf. [9, Ths. 2 and 3]), but we have found that our other applications fit nicely into more general settings which we shall study elsewhere (cf. [10] and [11]).

We thank the referee for useful comments and C. E. Burgess for discussions.

1. Property $(*, X, S)$, definitions, and notation. Suppose that $X$ is a closed subset of a 2 -sphere $S$ in $E^{3}$. We say that $S$ can be side approximated so as to miss $X$ if the following property is satisfied:

Property $(*, X, S) . \quad([15$, p. 467$])$. If $U$ is a component of $E^{3}-S$ and $\varepsilon$ is a positive number, then there are a polyhedral 2-sphere $S^{\prime}$, a finite collection $D_{1}, D_{2}, \cdots, D_{k}$ of mutually exclusive $\varepsilon$-disks lying in $S^{\prime}$, and a finite collection $E_{1}, E_{2}, \cdots, E_{n}$ of mutually exclusive $\varepsilon$-disks lying in $S$, such that

(1) There is an $\varepsilon$-homeomorphism from $S$ onto $S^{\prime}$,

(2) $S^{\prime}-\bigcup_{i=1}^{k} D_{i} \subset U$,

(3) $S \cap S^{\prime} \subset \bigcup_{i=1}^{n} E_{i}$, and

(4) $X \cap\left(\bigcup_{i=1}^{n} E_{i}\right)=\varnothing$.

If $(*, X, S)$ is satisfied, then $X$ is tame (i.e., $X$ lies on a tame 2-sphere in $E^{3}$ ) [19, Th. 6]. If $X$ has no degenerate components, then $X$ is tame if and only if $(*, X, S)$ is satisfied [8, Ths. 1.1 and 5.1].

If the property defined above holds for one component $U$ of $E^{3}-S$ though perhaps not for the other, we say that $(*, X, U)$ is satisfied. If $(*, X$, Int $S)$ is satisfied, then $X$ lies on the surface of a 3-cell in $E^{3}$ [21, Th. 1]. Our goal is to characterize sets satisfying $(*, X, \operatorname{Int} S)$ as those compact sets $X$ for which $(S \cup \operatorname{Int} S)-X$ is 1 ULC. A similar result will obviously hold for $U=\operatorname{Ext} S$.

REMARK. The definition that we have given of (*, $X, S)$ is the original formulation and not always the easiest to apply. If we wish to prove that $(*, X, \operatorname{Int} S)$ is satisfied, we shall use the definition we have stated. If we already know that $(*, X, \operatorname{Int} S)$ is satisfied and 
wish to apply that fact, we shall use the following formulation due to Lister. Lister proves [18, Th. 2] that the two definitions are equivalent.

Property $(*, X, \operatorname{Int} S)$ (alternative definition). If $\varepsilon>0$, then there are disjoint $\varepsilon$-disks $D_{1}, D_{2}, \cdots, D_{n}$ in $S-X$ and an $\varepsilon$-homeomorphism $h$ from $S$ onto a polyhedral sphere $h(S)$ in $E^{3}$ such that

(1) $h(S)-\bigcup_{i=1}^{n} \operatorname{Int} h\left(D_{i}\right) \subset \operatorname{Int} S$,

(2) $S-\bigcup_{i=1}^{n} \operatorname{Int} D_{i} \subset \operatorname{Ext} h(S)$, and

(3) $S \cap h(S)=\bigcup_{i=1}^{n}\left[\operatorname{Int} D_{i} \cap \operatorname{Int} h\left(D_{i}\right)\right]$.

RemarK. Property $(*, \varnothing, \operatorname{Int} S)$ is always satisfied $\left(\left[4\right.\right.$, Th. $\left.16^{\prime}\right]$ (original formulation) and [18, Th. 2] (alternative definition)).

We shall use the symbol $\rho$ for the Euclidean metric. If $A$ and $A^{\prime}$ are subsets of $E^{3}$ and $\varepsilon$ is a positive number, then we define

(1) $\operatorname{Diam} A=\sup \rho(x, y) \quad(x, y \in A)$,

(2) $\rho\left(A, A^{\prime}\right)=\inf \rho\left(a, a^{\prime}\right)\left(a \in A, a^{\prime} \in A^{\prime}\right)$, and

(3) $B\left(A, A^{\prime} ; \varepsilon\right)=\left\{a^{\prime} \in A^{\prime}\left\{\rho\left(a^{\prime}, A\right)<\varepsilon\right\}\right.$. (If $A^{\prime}=E^{3}$, we write $B\left(A, A^{\prime} ; \varepsilon\right)=B(A ; \varepsilon)$.)

The set $A$ is an $\varepsilon$-set if $\operatorname{Diam} A<\varepsilon . \quad$ A map $f: A \rightarrow E^{3}$ is called an $\varepsilon$-map if $f$ moves no point as far as $\varepsilon$. Let $\Delta$ and $I$ denote a standard disk or 2-simplex and a standard interval or 1-simplex, respectively. We say that $A$ is $1-\mathrm{LC}(0-\mathrm{LC})$ in $A^{\prime}$ at $x \in E^{3}$ if for each $\varepsilon>0$ there is a $\delta>0$ such that any map $f: \mathrm{Bd} \Delta \rightarrow B(x, A ; \delta)(f: \operatorname{Bd} I \rightarrow B(x, A ; \delta))$ has a continuous extension $f^{*}: \Delta \rightarrow B\left(x, A^{\prime} ; \varepsilon\right) \quad\left(f^{*}: I \rightarrow B\left(x, A^{\prime} ; \varepsilon\right)\right)$. Speaking loosely, $A$ is $1-\mathrm{LC}$ in $A^{\prime}$ at $x \in E^{3}$ if loops near $x$ in $A$ can be shrunk to a point near $x$ in $A^{\prime}$. We say that $A$ is 1-ULC (0-ULC) in $A^{\prime}$ if $A$ is $1-\mathrm{LC}(0-\mathrm{LC})$ in $A^{\prime}$ at each point $x$ of $\mathrm{Cl} A$ and if for each $\varepsilon>0$ the corresponding $\delta$ may be chosen independently of $x \in$ Cl $A$. If $A$ is 1-ULC in $A$, then we simply say that $A$ is 1-ULC. We say that $A$ is weakly $1-\mathrm{ULC}$ in $A^{\prime}$ if (speaking loosely again) small unknotted simple closed curves in $A$ bound small singular disks in $A^{\prime}$. (A simple closed curve in $E^{3}$ is said to be unknotted if it bounds a disk in $E^{3}$.) We leave it to the reader to formalize the definition of "weakly 1-ULC".

A crumpled cube $C$ is the union of a 2-sphere in $E^{3}$ and its interior in $E^{3}$. The 2-sphere is called the boundary of $C$, denoted $\mathrm{Bd} C$; and $C-\mathrm{Bd} C$ is called the interior of $C$, denoted Int $C$. Any homeomorphic image of $C$ is called a crumpled cube. A point $p \in \mathrm{Bd} C$ is said to be a piercing point of $C$ if there is an embedding $h: C \rightarrow E^{3}$ such that $h(\mathrm{Bd} C)$ can be pierced by a tame are at $h(p)$.

Most of our terminology not defined explicitly in this section is fairly standard and can be found in most of the references listed. 
See, for example, [2], [7], and [19].

2. A 1-ULC characterization of sets satisfying (*, $X$, Int $S)$. Our characterization will appear in two parts which we prove separately (Ths. 2.1 and 2.4).

Theorem 2.1. If $S$ is a 2-sphere in $E^{3}, X$ is a closed subset of $S$, and $(*, X$, Int $S)$ is satisfied, then $(S \cup \operatorname{Int} S)-X$ is 1-ULC.

Proof. Let $\varepsilon$ be a positive number. There is a $\delta$ in the range $0<\delta<\varepsilon / 6$ having the following property: if $S^{\prime}$ is a 2-sphere in $E^{3}$ such that there is a $\delta$-homeomorphism from $S$ onto $S^{\prime}$, then any $\delta$-set on $S^{\prime}$ lies on an $\varepsilon / 6$-disk on $S^{\prime}$. Let $f: \mathrm{Bd} \Delta \rightarrow(S \cup \operatorname{Int} S)-X$ be a map into a $\delta$-set in $(S \cup \operatorname{Int} S)-X$. In order to show that $(S \cup \operatorname{Int}$ $S)-X$ is 1 -ULC it suffices to show that $f$ is nullhomotopic $(f \sim 0)$ in an $\varepsilon$-subset of $(S \cup \operatorname{Int} S)-X$.

Because Int $S$ is 0-ULC [24, p. 66] and $S$ is an absolute neighborhood retract, there is a map $F: \mathrm{Bd} \Delta \times I \rightarrow(S \cup \operatorname{Int} S)-X$ such that $F_{0}=f, F_{1}: \mathrm{Bd} \Delta \rightarrow \operatorname{Int} S$, and Diam $F(\mathrm{Bd} \Delta \times I)<\delta$ (where $F_{t}$ : $\mathrm{Bd} \Delta \rightarrow E^{3}$ is defined by $\left.F_{t}(x)=F(x, t)\right)$. The map $F_{1}$ can be extended to a map $G: \Delta \rightarrow E^{3}$ such that $\operatorname{Diam} G(\Delta)<\delta$.

Since $(*, X, \operatorname{Int} S)$ is satisfied, there are disjoint $\delta$-disks $D_{1}, D_{2}$, $\cdots, D_{n}$ in $S-X$ and a $\delta$-homeomorphism $h$ from $S$ onto a polyhedral 2-sphere $h(S)$ in $E^{3}$ such that (1), (2), and (3) of the alternative definition for $(*, X$, Int $S)$ are satisfied and such that $h(S)$ separates $G(\operatorname{Bd} \Delta)=F_{1}(\operatorname{Bd} \Delta)$ from $X$.

The intersection of $G(\Delta)$ with $h(S)$ is a $\delta$-set. Hence by our choice of $\delta$, there is an $\varepsilon / 6$-disk $E$ on $h(S)$ which contains $G(\Delta) \cap h(S)$. By the Tietze extension theorem (see [7, Lemma 1] for details), there is. a map $G_{1}: \Delta \rightarrow E^{3}$ which agrees with $G$ on the component $K$ of

$$
\Delta-G^{-1}[G(\Delta) \cap h(S)]
$$

which contains $\mathrm{Bd} \Delta$ and takes $\Delta-K$ into $E$. Then

$$
G_{1}(\Delta) \cap S \subset \bigcup_{i=1}^{n} D_{i} .
$$

Again by the Tietze theorem there is a map $G_{2}: \Delta \rightarrow E^{3}$ which agrees with $G_{1}$ on the component $K^{\prime}$ of $\Delta-G_{1}^{-1}\left[G_{1}(\Delta) \cap \bigcup_{i=1}^{n} D_{i}\right]$ which contains $\mathrm{Bd} \Delta$ and takes $\Delta-K^{\prime}$ into $\bigcup_{i=1}^{n} D_{i}$.

Clearly $f \sim 0$ in $F(\mathrm{Bd} \Delta \times I) \cup G_{2}(\Delta)$. Thus it suffices to show that $F(\operatorname{Bd} \Delta \times I) \cup G_{2}(\Delta) \subset(S \subset \operatorname{Int} S)-X$ and that

$\operatorname{Diam}\left[F(\operatorname{Bd} \Delta \times I) \cup G_{2}(\Delta)\right]<\varepsilon$. 
That these conditions are satisfied is easily checked. This completes the proof of Theorem 2.1.

The second half of our characterization requires two lemmas. The first, Lemma 2.2, is a special case of [22, Lemma 2], which in turn is a special case of $[14, T h .2]$. We indicate a proof for Lemma 2.2 since it is a simple special case of the more difficult general results of [14]. Lemma 2.3, a consequence of Lemma 2.2, could be generalized considerably by appeal to these more general results. This is interesting when one notes that Theorem (0.4), a conjecture by Loveland, is simply the translation of Lemma 2.3, via Theorems 2.1 and 2.4 , into a statement about side approximations of 2-spheres.

Lemma 2.2. Let $B$ denote a closed subset of a disk $D$, let $S$ be a 2-sphere in $E^{3}$, and let $X$ be a subset of $S$ such that $(S \cup \operatorname{Int} S)-X$ is 1-ULC. Let $\varepsilon>0$ and a map $f: D \rightarrow S \cup \operatorname{Int} S$ be given. Then there is a map $f^{*}: D \rightarrow S \cup \operatorname{Int} S$ such that

$$
\begin{aligned}
& f^{*}(D-B) \subset(S \cup \operatorname{Int} S)-X, \\
& f^{*}|B=f| B, \text { and } \\
& \rho\left(f^{*}(x), f(x)\right)<\varepsilon \text { for each } x \in D .
\end{aligned}
$$

Indication of proof. One triangulates $D-B$ so that the mesh of the triangulation approaches zero very rapidly near $B$. Then using the facts that $\mathrm{Cl}((S \cup \operatorname{Int} S)-X)=(S \cup \operatorname{Int} S),(S \cup \operatorname{Int} S)-X$ is 0 ULC [24, p. 66], and $(S \cup \operatorname{Int} S)-X$ is 1 -ULC, one moves the image of the $0-, 1-$, and 2-skeletons of the triangulation away from $X$. Note that we do not require that $X$ be closed.

LEMMA 2.3. Let $S$ be a 2-sphere in $E^{3}$, and let $\left\{X_{i}\right\}_{i=1}^{\infty}$ be a family of closed subsets of $S$ such that, for each $i,(S \cup \operatorname{Int} S)-X_{i}$ is 1ULC. Then $(S \cup \operatorname{Int} S)-\bigcup_{i=1}^{\infty} X_{i}$ is 1-ULC.

Proof. Suppose $\varepsilon>0$ and $x \in S \cup \operatorname{Int} S$ given, and let $\Delta$ denote a disk. Since $(S \cup \operatorname{Int} S)-X_{1}$ is 1 -ULC, there is a $\delta>0$ such that each map $f: \operatorname{Bd} \Delta \rightarrow B\left(x,(S \cup \operatorname{Int} S)-X_{1} ; \delta\right)$ extends to a map

$$
f^{*}: \Delta \rightarrow B\left(x,(S \cup \operatorname{Int} S)-X_{1} ; \varepsilon\right) \text {. }
$$

Let $f: \mathrm{Bd} \Delta \rightarrow B\left(x,(S \cup \operatorname{Int} S)-\bigcup_{i=1}^{\infty} X_{i} ; \delta\right)$ be given, and let

$$
f_{1}: \Delta \rightarrow B\left(x,(S \cup \operatorname{Int} S)-X_{1} ; \varepsilon\right)
$$

be an extension of $f$. We now define positive numbers $\varepsilon_{1}, \varepsilon_{2}, \cdots$ and maps $f_{2}, f_{3}, \cdots$ inductively as follows:

Let 


$$
0<\varepsilon_{1}<1 / 2 \rho\left[f_{1}(\Delta), X_{1} \cup((S \cup \operatorname{Int} S)-B(x,(S \cup \operatorname{Int} S) ; \varepsilon))\right] .
$$

Let $f_{2}: \Delta \rightarrow(S \cup \operatorname{Int} S)-X_{2}$ be such that $f_{2}\left|\operatorname{Bd} \Delta=f_{1}\right| \mathrm{Bd} \Delta$ and

$$
\rho\left(f_{1}(p), f_{2}(p)\right)<\varepsilon_{1}
$$

for each $p \in \Delta$. Such a map exists by Lemma 2.2 .

For $i>1$, let $\varepsilon_{i}$ be in the range $0<\varepsilon_{i}<1 / 2 \min \left\{\varepsilon_{i-1}, \rho\left[f_{i}(\Delta), X_{i}\right]\right\}$. Let $f_{i+1}: \Delta \rightarrow(S \cup \operatorname{Int} S)-X_{i+1}$ be such that $f_{i+1}\left|\mathrm{Bd} \Delta=f_{i}\right| \mathrm{Bd} \Delta$ and $\rho\left(f_{i}(p), f_{i+1}(p)\right)<\varepsilon_{i}$ for each $p \in \Delta$. Again, at each stage such a map exists by Lemma 2.2.

Because $S \cup \operatorname{Int} S$ is a complete space, it follows that $f^{*}=\lim f_{i}$ exists, is continuous, extends $f$, and takes $\Delta$ into

$$
B\left(x,(S \cup \operatorname{Int} S)-\bigcup_{i=1}^{\infty} X_{i} ; \varepsilon\right) .
$$

Since $x$ is arbitrary and $\delta$ may be chosen independently of $x,(S \cup \operatorname{Int} S)-$ $\mathrm{U}_{i=1}^{\infty} X_{i}$ is 1 -ULC.

THEOREM 2.4. If $S$ is a 2-sphere in $E^{3}, X$ is a closed subset of $S$, and $(S \cup \operatorname{Int} S)-X$ is 1 -ULC, then $(*, X$, Int $S)$ is satisfied.

Proof. We first establish the theorem for the special case $X=S$ and then show how the proof can be altered to yield the general result. There are four steps in the special case.

1. There are disjoint disks $D_{1}, D_{2}, \cdots, D_{n}$ in $S$ and an $\varepsilon$-homeomorphism $h$ from $S$ onto a polyhedral sphere $h(S)$ in $E^{3}$ such that (1), (2), and (3) of the alternative definition of $(*, \varnothing$, Int $S)$ are satisfied and such that Diam $\left[D_{i} \cup h\left(D_{i}\right)\right]<\varepsilon$ for each $i$. This is because (*, $\varnothing$, Int $S$ ) is always satisfied.

2. By the Tietze Extension Theorem, there are maps $f_{1}, f_{2}, \cdots, f_{n}$ such that for each $i, f: D_{i} \rightarrow h\left(D_{i}\right) \cup \operatorname{Int} D_{i}, f_{i}$ agrees with $h$ on the component $K_{i}$ of $D_{i}-h^{-1}\left[h\left(D_{i}\right) \cap D_{i}\right]$ which contains $\mathrm{Bd} D_{i}$, and $f_{i}$ takes $D_{i}-K_{i}$ into $\operatorname{Int} D_{i}$.

3. By Lemma 2.2, since Int $S$ is 1-ULC, if $\delta>0$, then there is a map $g_{i}: D_{i} \rightarrow f_{i}\left(K_{i}\right) \cup B\left(D_{i}\right.$, Int $S$; $\left.\delta\right)$ which agrees with $f_{i}$ on

$$
K_{i}-f_{i}^{-1}\left[B\left(D_{i}, f_{i}(D) ; \delta\right)\right] .
$$

Thus we may assume that $g_{1}\left(D_{1}\right), g_{2}\left(D_{2}\right), \cdots, g_{n}\left(D_{n}\right)$ are disjoint singular disks in Int $S$ such that for each $i,(1) g_{i}\left(D_{i}\right)$ has no singularities near $g_{i}\left(\operatorname{Bd} D_{i}\right)$, (2) Diam $\left[g_{i}\left(D_{i}\right) \cup D_{i}\right]<\varepsilon$, and $(3) g_{i}\left(D_{i}\right) \cap h\left[S-\bigcup_{i=1}^{n} \operatorname{Int} D_{i}\right]=$ $g_{i}\left(\mathrm{Bd} D_{i}\right)$.

4. By Dehn's Lemma [23], each singular disk $g_{i}\left(D_{i}\right)$ may be replaced by a polyhedral disk $E_{i}$ in $\operatorname{Int} S$ such that 


$$
h\left[S-\bigcup_{i=1}^{n} \operatorname{Int} D_{i}\right] \cup \bigcup_{i=1}^{n} E_{i}
$$

is a polyhedral 2-sphere $S^{\prime}$ in Int $S$ and for each $i$, Diam $\left[E_{i} \cup D_{i}\right]<\varepsilon$. If $h^{\prime}$ is any homeomorphism from $S$ onto $S^{\prime}$ which extends

$$
h \mid S-\bigcup_{i=1}^{n} \operatorname{Int} D_{i},
$$

then $h^{\prime}$ is an $\varepsilon$-homeomorphism from $S$ into $\operatorname{Int} S$. Since $\varepsilon$ is arbitrary, this shows that $(*, S$, Int $S)$ is satisfied.

In the general case, we make the following changes:

$3^{\prime}$. Let $\left\{X_{i}\right\}_{i=1}^{\infty}$ be a family of tame finite graphs on $S$ such that $S-\bigcup_{i=1}^{\infty} X_{i}$ is totally disconnected. That such a family exists follows from [3, Th. 1]. If $A$ is an are in any $X_{i}$, then $(*, A, \operatorname{Int} S)$ is satisfied by [15, Th. 2]. Thus by Theorem 2.1, $(S \cup \operatorname{Int} S)-A$ is 1-ULC; and by Lemma 2.3, $(S \cup \operatorname{Int} S)-\left(\bigcup_{i=1}^{\infty} X_{i} \cup X\right)$ is 1 -ULC since $\bigcup_{i=1}^{\infty} X_{i} \cup X$ is a countable union of closed sets $A$ for which $(S \cup \operatorname{Int} S)-A$ is 1-ULC. Hence by Lemma 2.2, if $\delta>0$, there is a map $g_{i}: D_{i} \rightarrow$ $f_{i}\left(K_{i}\right) \cup B\left(D_{i},(S \cup \operatorname{Int} S)-\left(\bigcup_{i=1}^{\infty} X_{i} \cup X\right) ; \delta\right)$ which agrees with $f_{i}$ on $K_{i}-f_{i}^{-1}\left[B\left(D_{i}, f_{i}\left(D_{i}\right) ; \delta\right)\right]$. Thus we may assume that

$$
g_{1}\left(D_{1}\right), g_{2}\left(D_{2}\right), \cdots, g_{n}\left(D_{n}\right)
$$

are disjoint singular disks in $(S \cup \operatorname{Int} S)-X$ which satisfy (1), (2), and (3) of Step 3 above and such that for each $i, g_{i}\left(D_{i}\right) \cap S$ is a 0 dimensional subset of Int $D_{i}$.

$4^{\prime}$. The set $g_{i}\left(D_{i}\right) \cap S$, being a closed 0 -dimensional subset of Int $D_{i}-X$, can be covered by a finite collection $E_{i 1}, E_{i 2}, \cdots, E_{i n_{i}}$ of disjoint disks in Int $D_{i}-X$. Again by Dehn's Lemma, each singular disk $g_{i}\left(D_{i}\right)$ may be replaced by a polyhedral disk $E_{i}$ such that

$$
h\left[S-\bigcup_{i=1}^{n} \operatorname{Int} D_{i}\right] \cup \bigcup_{i=1}^{n} E_{i}
$$

is a polyhedral 2-sphere $S^{\prime}$ in $E^{3}$ and for each $i, E_{i} \cap S \subset \bigcup_{j=1}^{n_{i}} E_{i j}$ and Diam $\left[E_{i} \cup D_{i}\right]<\varepsilon$. But since $\varepsilon$ is arbitrary, $S^{\prime}$ and the disks $\left\{E_{i}\right\}$ and $\left\{E_{i j}\right\}$ are precisely what one needs in order to show that (*, $X, \operatorname{Int} S)$ is satisfied, where in this case we apply the original definition.

We now show that the hypotheses of Theorem 2.4 can be weakened. Our goal is Theorem 2.6. In the proof of Theorem 2.6 we need to know that a polyhedral disk in $E^{3}$ whose boundary lies in the interior of a particular 2-sphere in $E^{3}$ (possibly a wild 2-sphere in $E^{3}$ ) can, in a particularly nice way, almost be cut off inside the 2-sphere. This fact, stated precisely in Lemma 2.5, will be apparent to those thoroughly familiar with the methods of [5]. However, following the suggestion of the referee, we give a proof which does not rely on the methods of 
[5] and depends only on Bing's Side Approximation Theorem [4]. The brief outline with which the proof of Lemma 2.5 begins is due to the referee and had also been suggested to the author by C. E. Burgess.

\section{LEMma 2.5. Suppose that}

$S$ is a 2-sphere in $E^{3}$;

$U$ is a component of $E^{3}-S$;

$D$ is a polyhedral disk in $E^{3}$ with $\mathrm{Bd} D \subset U$;

$R$ is a disk in $S$ with $D \cap S \subset R$;

$0<\varepsilon<\rho(\mathrm{Bd} D, S) ;$ and

$K$ is the the component of $D-S$ which contains $\mathrm{Bd} D$. Then there is a map $h: D \rightarrow(D \cap U) \cup B(R, \mathrm{Cl} U ; \delta)$ such that

$$
\begin{aligned}
& h \mid K-B(R ; \varepsilon)=\text { identity } \\
& h(D) \cap S \text { is 0-dimensional; and } \\
& h \mid D-h^{-1}(S) \text { is a homeomorphism. }
\end{aligned}
$$

Proof. The plan is the following: Let $S_{1}$ be a side approximation to $S$ from $U$ sufficiently close to $S$. Cut $D_{0}=D$ off on $S_{1}$ to get a new polyhedral disk $D_{1}$. Again let $S_{2}$ be a side approximation to $S$ from $U$ sufficiently close to $S$ (much closer than $S_{1}$ ). Cut $D_{1}$ off on $S_{2}$ to obtain a new polyhedral disk $D_{2}$. Repeat this procedure. If proper care is taken in the process, then $h(D)$ may be taken to be $\lim D_{i}$. In the next few paragraphs we explain in turn how each of the conclusions of the lemma can be obtained by this procedure.

Associating a continuous function $h$ with $\lim D_{i}$. Cutting $D_{i-1}$ off on $S_{i}$ can be realized as a process of removing from $D_{i-1}$ finitely many disjoint disks ${ }^{i} \Delta_{1}, \cdots,{ }^{i} \Delta_{r}$ in Int $D_{i-1}$ and replacing them by disjoint disks ${ }^{i} \Delta_{1}^{\prime}, \cdots,{ }^{i} \Delta_{r}^{\prime}$ near $S_{i}$. Thus there is a natural, though nonunique, way of defining a homeomorphism $h_{i}: D_{i-1} \rightarrow D_{i}: h_{i}$ differs from the identity only in that it takes a disk ${ }^{i} \Delta_{j}$ removed in the cutting process to its replacement ${ }^{i} \Delta_{j}^{\prime}$ near $S_{i}$. A function

$$
h=\lim h_{i} \cdots h_{2} h_{1}: D \rightarrow \lim D_{i}
$$

will therefore exist and be continuous provided the size of the disks removed and the size of their replacements converge to 0 rapidly enough with $i$. Suppose that to this end we want the disks $\left\{{ }^{i+1} \Delta_{j}\right\}$ and $\left\{{ }^{i+1} \Delta_{j}^{\prime}\right\}$ to have diameter less than $\alpha$. This can be accomplished by using caution in the adjustments which change $D_{i-1}$ to $D_{i}$. The procedure is tedious but straightforward. The reader may wish to omit a reading of the details which follow:

Choose $\beta, 0<\beta<\alpha$, such that $\beta$-sets in a $\beta$-approximation $S^{\prime}$ to $S$ lie in $\alpha$-disks in $S^{\prime}$. 
Choose $\gamma, 0<\gamma<\beta / 3$, such that $\gamma$-sets in a $\gamma$-approximation $S^{\prime \prime}$ to $S$ lie in $\beta / 3$-disks in $S^{\prime \prime}$.

Choose $\delta, 0<\delta<\gamma$, such that $\delta$-sets in a $\delta$-approximation $D_{i-1}^{\prime}$ to $D_{i-1}$ lie in $\gamma$-disks in $D_{i-1}^{\prime}$.

Choose a $\delta$-side approximation $S_{i}$ to $S$ from $U$ with associated $\delta$-disks $E_{1}, \cdots, E_{n}$ on $S_{i}$ and $F_{1}, \cdots, F_{m}$ on $S$ containing the intersections of $S_{i}$ with $S$.

Adjust $D_{i-1}$ slightly near $S_{i}$ (by a $\delta$-adjustment) so that the adjusted $D_{i-1}$ misses $\cup E_{j}$ and is in general position with respect to $S_{i}$. Call the adjusted $D_{i-1}$ by the same name as the original.

Cut $D_{i-1}$ off on $S_{i}$ to form $D_{i}$. This cutting removes disjoint disks $\left\{{ }^{i} \Delta_{j}\right\}$ from Int $D_{i-1}$ and replaces them by disks $\left\{{ }^{i} \Delta_{j}^{\prime}\right\}$ near $S_{i}$.

We now show that $D_{i} \cap S$ can be covered by a finite collection of disjoint $\beta$-disks in Int $D_{i}$. Since $\left[D_{i-1}\right.$ (adjusted) $] \cap\left(\cup E_{j}\right)=\varnothing$, the disk-with-holes $K_{i}=\operatorname{cl}\left(D_{i-1}-\cup^{i} \Delta_{j}\right)$ intersects $S$ only in Int $K_{i}$ and if care has been taken, also only in $U F_{j}$. From this latter fact, each component of $K_{i} \cap S$ has diameter less than $\delta$. Hence by our choice of $\delta, K_{i} \cap S$ can be covered by a finite collection of disjoint $\gamma$-disks in $D_{i-1}$, each of which has boundary in Int $K_{i}$. The image of each such disk under $h_{i}: D_{i-1} \rightarrow D_{i}$ has diameter less than

$$
\gamma+2 \beta / 3<\beta \text {. }
$$

A disk ' $\Delta_{j}^{\prime}$ is either contained in one of these $\beta$-disks or intersects none of them. In the latter case, note that ${ }^{i} \Delta_{j}^{\prime}$ is very close homeomorphically to some disk $H$ in $S_{i}$ such that $\mathrm{Bd} H \cap\left(\cup E_{k}\right)=\varnothing$. If ${ }^{i} \Delta_{j}^{\prime}$ is close enough to $H$, then ${ }^{i} \Delta_{j}^{\prime} \cap S$ is covered in a natural way by $\beta$-disks in Int ${ }^{i} \Delta_{j}^{\prime}$ associated with the $\delta$-disks $\left\{E_{k} \mid E_{k} \subset \operatorname{Int} H\right\}$. We thus obtain finally a finite collection of disjoint $\beta$-disks in $\operatorname{Int} D_{i}$ covering $D_{i} \cap S$.

If $S_{i+1}$ is a $\beta$-approximation to $S$ that intersects $D_{i}$ only in the finite union of these disjoint $\beta$-disks, then the disks $\left\{{ }^{i+1} \Delta_{j}\right\}$ will be of diameter less than $\beta(<\alpha)$ and the disks $\left\{{ }^{i+1} \Delta_{j}^{\prime}\right\}$ of diameter less than $\alpha$ by our choice of $\beta$.

These considerations show that the approximations $S_{i}$ and disks $D_{i}$ may be chosen so that $h=\lim h_{i} \cdots h_{2} h_{1}$ automatically exists and is continuous.

Making $h \mid K-B(R ; \varepsilon)$ the identity. One simply needs to keep all the spheres $S_{i}$ away from some continuum in $K$ which contains $K-B(R ; \varepsilon)$.

Keeping $h(D)$ in $(D \cap U) \cup B(R, \mathrm{Cl} U ; \varepsilon)$. Note that

$$
h(D)=\lim D_{i} \subset \lim \left(S_{i} \cup U\left(\mathbf{S}_{i}\right)\right)=\mathrm{Cl} U
$$

(where $U\left(\mathbf{S}_{i}\right)$ is the complementary domain of $S_{i}$ which "almost coin- 
cides" with $U)$. Then if one keeps all of the disks $\left\{{ }^{i} \Delta_{j}^{\prime}\right\}$ in $B(R, \varepsilon / 2)$, which one may clearly do, it follows immediately that

$$
h(D) \subset(D \cap U) \cup B(R ; \varepsilon) \text {. }
$$

Making $h(D) \cap \mathrm{S}$ 0-dimensional. As we showed in the first part of this proof, by being careful at stage $i$ we can require at stage $i+1$ that $D_{i} \cap \mathrm{S}$ be covered by a collection of disjoint $\alpha$-disks in $S$. One may then require that all of the disks $\left\{{ }^{k} \Delta_{j}^{\prime}\right\}(k \geqq i+1)$ lie in an arbitrary neighborhood of the union of these $\alpha$-disks. It follows then that $h(D) \cap S$ also lies very close to the union of these $\alpha$-disks, hence has no component of diameter much larger than $\alpha$. If our $\alpha$ 's converge to 0 , then $h(D) \cap S$ will be 0 -dimensional.

Making $h \mid D-h^{-1}(S)$ a homeomorphism. This is automatic since each $h_{i}$ is a homeomorphism and each point mapped into $U$ has a neighborhood moved by only finitely many of the maps $h_{i}$.

THEOREM 2.6. If $S$ is a 2-sphere in $E^{3}, X$ is a closed subset of $S$, and Int $S$ is weakly 1-ULC in $(S \cup \operatorname{Int} S)-X$, then $(S \cup \operatorname{Int} S)-X$ is 1-ULC.

Proof. By Theorem 2.1, it suffices to show that $(*, X$, Int $S)$ is satisfied. The proof is like that of the general case of Theorem 2.4 except that Lemma 2.5 allows us to alter the second step so that our weaker hypothesis is applicable in the third step. The first and fourth steps remain unchanged.

$2^{\prime \prime}$. There are subdisks $E_{1}, E_{2}, \cdots, E_{n}$ of $D_{1}, D_{2}, \cdots, D_{n}$ respectively such that for each $i, h\left(D_{i}\right) \cap D_{i} \subset E_{i} \subset \operatorname{Int} D_{i}$. Choose $\alpha>0$ such that $\alpha<1 / 3 \rho\left[E_{i},\left(S-D_{i}\right) \cup h\left(S-D_{i}\right)\right]$ and such that

$$
\operatorname{Diam}\left[h\left(D_{i}\right) \cup B\left(D_{i} ; \alpha\right)\right]<\varepsilon
$$

for each $i$. By Lemma 2.5, there are maps $f_{1}, f_{2}, \cdots, f_{n}$ such that for each $i, f_{i}: D_{i} \rightarrow h\left(D_{i}\right) \cup B\left(E_{i}, S \cup \operatorname{Int} S ; \alpha\right), f_{i}$ agrees with $h$ on the component of $D_{i}-h^{-1}\left[B\left(D_{i}, S \cup \operatorname{Int} S ; \alpha\right)\right]$ which contains $\operatorname{Bd} D_{i}, f_{i}\left(D_{i}\right) \cap S$ is a 0 -dimensional subset of Int $D_{i}$, and $f_{i} \mid D_{i}-f_{i}^{-1}(S)$ is a homeomorphism. Define $f: S \rightarrow E^{3}$ piecewise by $f\left|S-\bigcup_{i=1}^{n} D_{i}=h\right| S-\bigcup_{i=1}^{n} D_{i}$ and $f \mid D_{i}=f_{i}$.

$3^{\prime \prime}$. Choose $\beta$ such that

$$
0<\beta<1 / 3 \rho\left(f\left(D_{i}\right) \cap D_{i},\left(S-D_{i}\right) \cup f\left(S-D_{i}\right)\right)
$$

and such that $\operatorname{Diam}\left[f\left(D_{i}\right) \cup B\left(D_{i} ; \beta\right)\right]<\varepsilon$ for each $i$. Choose $\gamma$, using 
the hypothesis that $\operatorname{Int} S$ is weakly $1-U L C$ in $(S \cup \operatorname{Int} S)-X$, such that each unknotted $\gamma$-simple closed curve in Int $S$ bounds a singular $\beta / 2$-disk in $(S \cup \operatorname{Int} S)-X$. We require that $0<\gamma<\beta / 2$. Let $K_{i}$ denote the component of $D_{i}-f^{-1}(S)$ which contains $\operatorname{Bd} D_{i}$. Since $f\left(D_{i}\right) \cap S$ is 0 -dimensional, there is a finite collection $J_{1}, J_{2}, \cdots, J_{k}$ of disjoint simple closed curves in $K_{i}$ such that $\bigcup_{j=1}^{k} J_{j}$ separates $\operatorname{Bd} D_{i}$ from $f^{-1}(S)$ in $D_{i}$ and such that each $f\left(J_{j}\right)$ is a $\gamma$-loop in a $\gamma$ neighborhood of the set $f\left(D_{i}\right) \cap D_{i}$. We assume that no proper subcollection of $J_{1}, J_{2}, \cdots, J_{k}$ separates $\mathrm{Bd} D_{i}$ from $f^{-1}(S)$ in $D_{i}$. Because $f \mid D_{i}-f^{-1}(S)$ is a homeomorphism and $K_{i} \subset D_{i}-f^{-1}(S)$, it follows from Dehn's Lemma [23] that each $f\left(J_{j}\right)$ is an unknotted $\gamma$-simple closed curve in Int $S$. Hence by the way $\gamma$ was chosen, there is a map $g_{i}: D_{i} \rightarrow(S \cup \operatorname{Int} S)-X$ such that $g_{i}$ agrees with $f$ on that component of $K_{i}-\bigcup_{j=1}^{k} J_{j}$ which contains $\mathrm{Bd} D_{i}$ and such that $g_{i}$ takes the disk in $D_{i}$ bounded by $J_{j}$ into a $\beta / 2$-subset of $(S \cup \operatorname{Int} S)-X$. Define $g: S \rightarrow E^{3}$ piecewise by $g\left|S-\bigcup_{i=1}^{n} D_{i}=f\right| S-\bigcup_{i=1}^{n} D_{i}$ and $g \mid D_{i}=g_{i}$.

Now proceed as in Step $3^{\prime}$ to choose $\left\{X_{i}\right\}_{i=1}^{\infty}$. Apply Lemma 2.3 only to the set $(S \cup \operatorname{Int} S)-\bigcup_{i=1}^{\infty} X_{i}$ to find this set to be 1-ULC. Then relative to some fixed $\delta>0$, choose $h_{i}: D_{i} \rightarrow(S \cup \operatorname{Int} S)-\bigcup_{i=1}^{\infty} X_{i}$ as $g_{i}$ was chosen in Step $3^{\prime}$. Require further that $\rho\left(g(x), h_{i}(x)\right)<\delta$ for each $x \in D_{i}$. Now for $0<\delta<\rho\left(g\left(D_{i}\right), X\right)$ and for $\delta$ chosen otherwise sufficiently small, it follows as in Step $3^{\prime}$ that

$$
h_{1}\left(D_{1}\right), h_{2}\left(D_{2}\right), \cdots, h_{n}\left(D_{n}\right)
$$

are disjoint singular disks in $(S \cup \operatorname{Int} S)-X$ which satisfy (1), (2) and (3) of Step 3 (special case) and such that for each $i, h_{i}\left(D_{i}\right) \cap S$ is a 0 -dimensional subset of $\operatorname{Int} D_{i}$.

The proof can now be completed as in Step $4^{\prime}$.

\section{Proofs of Theorems $(0.1)-(0.4)$.}

Proof of Theorem (0.1). This is essentially the content of the special case of Theorem 2.4 which we proved first. Since $E^{3}-S$ is 1-ULC, Theorem 2.4 and its counterpart for sets satisfying $(*, X, \operatorname{Ext} S)$ imply that $(*, S, S)$ is satisfied. That is, $S$ can be homeomorphically approximated from each of its complementary domains. Thus $S$ is tame [1, Th. 2.2].

Proof of Theorem (0.2). Suppose the point $p$ is a piercing point of $C$, and $h: C \rightarrow E^{3}$ is an embedding so that $\mathrm{Bd} h(C)$ can be pierced 
by a tame arc at $h(p)$. Then by $(15$, see Th. 11], $(*, h(p)$, Int $h(C))$ is satisfied. By [18, Th. 7] (or from Theorems 2.1 and 2.4 of this paper) $(*, p$, Int $C)$ is satisfied. Thus by Theorem $2.1, C-p$ is 1 ULC.

Conversely, if $C-p$ is 1-ULC, let $h: C \rightarrow E^{3}$ be an embedding such that $\mathrm{Bd} h(C)$ is tame from $E^{3}-h(C)$ ([16], [17], or [12]). Then $h(C)-h(p)$ is 1-ULC, and clearly $\left(*, h(p), E^{3}-h(C)\right)$ is satisfied. Hence by Theorem 2.4, $(*, h(p), h(\mathrm{Bd} C))$ is satisfied. Therefore by [15, see Th. 11], $p$ is a piercing point of $C$.

Proof of Theorem (0.3). Since the statement of the theorem is symmetric in $S$ and $S^{\prime}$, we assume that $(*, X, S)$ is satisfied and prove under this assumption that $\left(*, X, S^{\prime}\right)$ is satisfied. By the compactness of $S^{\prime}$, the uniform nature of $E^{3}$, and Theorems 2.1 and 2.4 , it is sufficient to show that the sets $\left(S^{\prime} \cup \operatorname{Int} S^{\prime}\right)-X$ and $\left(S^{\prime} \cup\right.$ Ext $\left.S^{\prime}\right)-X$ are 1-LC at each point $x \in S^{\prime}$. If $x \in S^{\prime}-X$, both sets are clearly 1LC at $x$. If $x \in X$, then since $S$ and $S^{\prime}$ share an open set containing $X$, both spheres are locally the same at $x$. Thus clearly $\left(S^{\prime} \cup \operatorname{Int} S^{\prime}\right)-X$ and $\left(S^{\prime} \cup\right.$ Ext $\left.S^{\prime}\right)-X$ are $1-\mathrm{LC}$ at $x$ if and only if $(S \cup \operatorname{Int} S)-X$ and $(S \cup \operatorname{Ext} S)-X$ are $1-\mathrm{LC}$ at $x$. This completes the proof.

Proof of Theorem (0.4). The set $(S \cup \operatorname{Int} S)-X$ is 1-ULC by Theorem 2.1 and Lemma 2.3. Thus since $X$ is closed $(*, X, \operatorname{Int} S)$ is satisfied by Theorem 2.4. Similarly $(*, X$, Ext $S)$ is satisfied.

REMARK. [6, Th. 2.1] can be shown to be a special case of Theorem (0.4). Theorems 8, 9, and 10 of [20] are stated for closed subsets $G$ of a 2-sphere $S$ in $E^{3}$ such that the diameters of the components of $G$ have a positive lower bound. It follows from Theorem 2.4 that the restriction that the diameters of $G$ have a positive lower bound is unnecessary. That is, slight modifications of Loveland's proofs yield the following theorem:

THEOREM. If $G$ is a compact subset of a 2-sphere $S$ in $E^{3}$ and if any of the following conditions is satisfied, then $G$ lies on a tame 2-sphere in $E^{3}$.

(i) The set $G$ can be locally spanned in each complementary domain of $S$ on tame simple closed curves.

(ii) The set $G$ can be locally spanned in each component of $E^{3}-S$ missing Cantor sets.

(iii) The sphere $S$ can be locally spanned at each point of $G$ from each complementary domain of $S$. 
Similarly, a version of Eaton's theorem on strongly locally spherical spheres [13, Th. 3] can be stated and proved using Theorem 2.4 and modifications of Eaton's proof.

THEOREM. If $G$ is a compact subset of a 2-sphere $S$ in $E^{3}$ such that $S$ is strongly locally spherical at each point of $G$, then $G$ lies on a tame 2-sphere.

\section{REFERENCES}

1. R. H. Bing, Conditions under which a surface in $E^{3}$ is tame, Fund. Math. 47 (1959), 105-139.

2. - A surface is tame if its complement is 1-ULC, Trans. Amer. Math. Soc. 101 (1961), 294-305.

3. — Each disk in $E^{3}$ contains a tame arc, Amer. J. Math. 84 (1962), 583-590.

4. _ - Approximating surfaces from the side, Ann. of Math. (2) 77 (1963), 145192.

5. _ـ, Pushing a 2-sphere into its complement, Michigan Math. J. 11 (1964), 3345 .

6. Improving the side approximation theorem, Trans. Amer. Math. Soc. 116 (1965), 511-525.

7. C. E. Burgess, Characterizations of tame surfaces in $E^{3}$, Trans. Amer. Math. Soc. 114 (1965), 80-97.

8. J. W. Cannon, Characterization of taming sets on 2-spheres, Trans. Amer. Math. Soc. 147 (1970), 289-299.

9. Characterizations of sets which can be missed by side approximations of spheres, Noticer Amer. Math. Soc. 16 (1969), 174.

10. - Singular side approximations for 2-spheres in $E^{3}$ (in preparation)

11. *-taming sets for crumpled cubes (in preparation)

12. R. J. Daverman, A new proof for the Hosay-Lininger Theorem about crumpled cubes, Proc. Amer. Math. Soc. 23 (1969), 52-54.

13. W. T. Eaton, A note about locally spherical spheres, Canad. J. Math. 21 (1969), 1001-1003.

14. S. Eilenberg and R. L. Wilder, Uniform local connectedness and contractibility, Amer. J. Math. 64 (1942), 613-622.

15. David S. Gillman, Side approximation, missing an arc, Amer. J. Math. 85 (1963), 459-476.

16. Norman Hosay, The sum of a real cube and a crumpled cube is $S^{3}$, Notices Amer. Math. Soc. 10 (1963) 666.

17. L. L. Lininger, Some results on crumpled cubss, Trans. Amer. Math. Soc. 118 (1965), 534-549.

18. F. M. Lister, Simplifying intersections of disks in Bing's side approximation theorem, Pacific J. Math. 22 (1967), 281-295.

19. L. D. Loveland, Tame subsets of spheres in $E^{3}$, Pacific J. Math. 19 (1966), 489517.

20. Tame surfaces and tame subsets of spheres in $E^{3}$, Trans. Amer. Math. Soc. 123 (1966), 355-368.

21. Sufficient conditions for a closed set to lie on the boundary of a 3-cell, Proc. Amer. Math. Soc. 19 (1968), 649-652.

22. D. R. McMillan, Jr., Some topological properties of piercing points, Pacific J. Math. 22 (1967), 313-322.

23. C. D. Papakyriakopoulos, On Dehn's lemma and the asphericity of knots, Ann. of 
Math. (2) 66 (1957), 1-26.

24. R. L. Wilder, Topology of manifolds, Amer. Math. Soc. Colloquium Publications 32 (1949).

Received September 30, 1969, and in revised form December 5, 1969. These results were obtained while the author was a National Science Foundation Graduate Fellow at the University of Utah.

UNIVERSITY OF UTAH

UTAH State UNIVERSity

UNIVERSITY OF WISCONSIN 


\title{
PACIFIC JOURNAL OF MATHEMATICS
}

\author{
EDITORS
}

\author{
H. SAMELSON \\ Stanford University \\ Stanford, California 94305 \\ RichaRd PIERCE \\ University of Washington \\ Seattle, Washington 98105
}

J. DugundJI

Department of Mathematics

University of Southern California

Los Angeles, California 9.0007

RICHARD ARENS

University of California

Los Angeles, California 9.0024

\section{ASSOCIATE EDITORS}

E. F. BeCKenBACH
B. H. NeumanN

F. WoLE

K. YoSHIDA

\section{SUPPORTING INSTITUTIONS}

\author{
UNIVERSITY OF BRITISH COLUMBIA \\ CALIFORNIA INSTITUTE OF TECHNOLOGY \\ UNIVERSITY OF CALIFORNIA \\ MONTANA STATE UNIVERSITY \\ UNIVERSITY OF NEVADA \\ NEW MEXICO STATE UNIVERSITY \\ OREGON STATE UNIVERSITY \\ UNIVERSITY OF OREGON \\ OSAKA UNIVERSITY \\ UNIVERSITY OF SOUTHERN CALIFORNIA
}

\author{
STANFORD UNIVERSITY \\ UNIVERSITY OF TOKYO \\ UNIVERSITY OF UTAH \\ WASHINGTON STATE UNIVERSITY \\ UNIVERSITY OF WASHINGTON \\ AMERICAN MATHEMATICAL SOCIETY \\ CHEVRON RESEARCH CORPORATION \\ TRW SYSTEMS \\ NAVAL WEAPONS CENTER
}

The Supporting Institutions listed above contribute to the cost of publication of this Journal, but they are not owners or publishers and have no responsibility for its content or policies.

Mathematical papers intended for publication in the Pacific Journal of Mathematics should be in typed form or offset-reproduced, (not dittoed), double spaced with large margins. Underline Greek letters in red, German in green, and script in blue. The first paragraph or two must be capable of being used separately as a synopsis of the entire paper. The editorial "we" must not be used in the synopsis, and items of the bibliography should not be cited there unless absolutely necessary, in which case they must be identified by author and Journal, rather than by item number. Manuscripts, in duplicate if possible, may be sent to any one of the four editors. Please classify according to the scheme of Math. Rev. Index to Vol. 39. All other communications to the editors should be addressed to the managing editor, Richard Arens, University of California, Los Angeles, California, 90024.

50 reprints are provided free for each article; additional copies may be obtained at cost in multiples of 50 .

The Pacific Journal of Mathematics is published monthly. Effective with Volume 16 the price per volume (3 numbers) is $\$ 8.00$; single issues, $\$ 3.00$. Special price for current issues to individual faculty members of supporting institutions and to individual members of the American Mathematical Society: $\$ 4.00$ per volume; single issues $\$ 1.50$. Back numbers are available.

Subscriptions, orders for back numbers, and changes of address should be sent to Pacific Journal of Mathematics, 103 Highland Boulevard, Berkeley, California, 94708.

PUBLISHED BY PACIFIC JOURNAL OF MATHEMATICS, A NON-PROFIT CORPORATION

Printed at Kokusai Bunken Insatsusha (International Academic Printing Co., Ltd.), 7-17, Fujimi 2-chome, Chiyoda-ku, Tokyo, Japan. 


\section{Pacific Journal of Mathematics}

\section{Vol. 34, No. $2 \quad$ June, 1970}

Shair Ahmad, On the oscillation of solutions of a class of linear fourth order

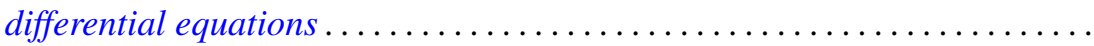

Leonard Asimow and Alan John Ellis, Facial decomposition of linearly

compact simplexes and separation of functions on cones ..............

Kirby Alan Baker and Albert Robert Stralka, Compact, distributive lattices of

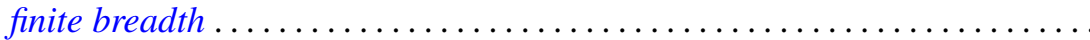

James W. Cannon, Sets which can be missed by side approximations to

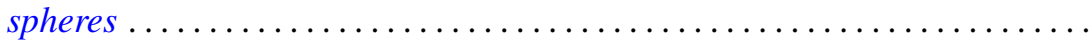

Prem Chandra, Absolute summability by Riesz means .................. 335

Francis T. Christoph, Free topological semigroups and embedding topological semigroups in topological groups....

Henry Bruce Cohen and Francis E. Sullivan, Projecting onto cycles in smooth, reflexive Banach spaces.................................

John Dauns, Power series semigroup rings .......................

Robert E. Dressler, A density which counts multiplicity ................

Kent Ralph Fuller, Primary rings and double centralizers ................

Gary Allen Gislason, On the existence question for a family of products.......

Alan Stuart Gleit, On the structure topology of simplex spaces .............

William R. Gordon and Marvin David Marcus, An analysis of equality in

certain matrix inequalities. $I \ldots \ldots \ldots \ldots \ldots \ldots \ldots \ldots$

Gerald William Johnson and David Lee Skoug, Operator-valued Feynman

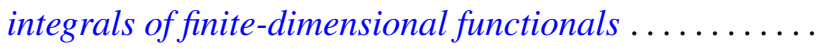

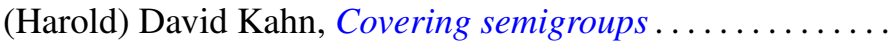

Keith Milo Kendig, Fibrations of analytic varieties

Norman Yeomans Luther, Weak denseness of nonatomic measures on perfect,

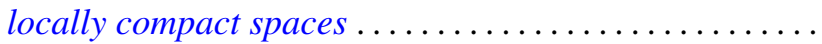

Guillermo Owen, The four-person constant-sum games; Discriminatory solutions on the main diagonal ...

Stephen Parrott, Unitary dilations for commuting contractions

Roy Martin Rakestraw, Extremal elements of the convex cone $A_{n}$ of

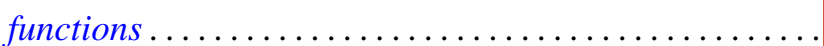

Peter Lewis Renz, Intersection representations of graphs by

William Henry Ruckle, Representation and series summability of complete

biorthogonal sequences.

F. Dennis Sentilles, The strict topology on bounded sets ...

Saharon Shelah, A note on Hanf numbers ...

Harold Simmons, The solution of a decision problem for several classes of rings. . .

Kenneth S. Williams, Finite transformation formulae involving the Legendre 